\title{
ANALYSIS OF ANTHOCNET AND AODV PERformanCE USING NS2
}

\author{
Y. Lakshmi Prasanna ${ }^{1}$ and Dr. P. Chenna Reddy ${ }^{2}$ \\ ${ }^{1}$ Department of Computer Science and Engineering, JNTUACEP, Pulivendula \\ ${ }^{2}$ Professor of CSE Department, JNTUACEP, Pulivendula
}

\begin{abstract}
Adhoc wireless multi-hop Networks (AHWMNs) are built with wireless nodes arranged in an adhoc manner. Every node can forward packets and also acts as a source. AODV establishes a path to receiver when it is needed by the sender and is on the standardization process of Internet engineering task force. AntHocNetwhich is based on ants foraging behavior, includes reactive and proactive mechanisms. AntHocNet builds the path as per the requirement of source and maintains until the end of communication session. In this paper performance of $A O D V$ and AntHocNetare analyzed at different parameters like data rates, pause times, and speed. Metrics Packet delivery ratio, Loss rate, End to End delay, jitter, and throughput are evaluated at different simulation times. Simulation is performed using network simulator NS-2.34 and $802.11 b$ is the MAC protocol.
\end{abstract}

\section{KEYWORDS}

Performance, Analysis, AHWMN, AODV, AntHocNet.

\section{INTRODUCTION}

An Adhoc wireless multi-hop networks (AHWMNs) [1] don't have any fixed infrastructure. Node can act as a source or destination or a router. Every node is independent and doesn't have any central coordinator. Nodes can leave the network at any time. In AHWMNS routing protocols are classified into reactive, proactive and hybrid routing protocols. Reactive routing protocols find the route when source needs to send data to destination. The route establishment is done by flooding the packets throughout the network. Proactive routingprotocols maintain the latest routing information at each node. AntHocNet is bio-inspired routing protocol which is a hybrid routing protocol. It follows reactive route setup and proactive path maintenance.

Bio-inspired routing protocols solve the routing problem in AHWMNs. They are based on Ant colony optimization (ACO). During the ants search for food when ants found the source of food they return to the place where they have started. Ants release a chemical called as pheromone on the path. By using different routes many ants will travel to the same food source. The ants which travel the minimum distant path will lay more pheromone in that path to help the other ants to move in that path. More ants are influenced by this pheromone. Ants communicate indirectly using a phenomenon known as stigmergy. In this an agent will leave signals for others and other agents sense them. This mode of communication is local where in simple agents interact locally without global information.

AntHocNet is a hybrid algorithm [3]that contains both reactive and proactive elements. AntHocNet gathers the routing information only when the source and destination are involved in DOI: $10.5121 /$ ijci.2015.4209 
a communication session and it is proactive means, it tries to maintain and improve information about existing paths while the session is going on. Routing information is stored in pheromone tables.The control packets and data packets are sent in a stochastic way using these tables.

In reactive route setup process when source node wants to connect with destination node, it checks it's routing table for the address of destination node. If it is not available then broadcast messages are flooded through the whole network. These are called as reactive forward ants. The intermediate node that receives the broadcast message will broadcast that if it is unaware of destination node otherwise it will unicast the message. The reactive forward ants will keep the list of nodes through which they have passed. When they are received by the destination then they will be converted into reactive backward ant. It follows the same path which the forward ant has been used and it also collects the quality information about each link of the path and updates at every intermediate node and source.

In proactive path maintenance two phases are there. They are pheromone diffusion and proactive ant sampling. In pheromone diffusion nodes periodically broadcasts best quality information. Using this information all nodes will calculate new pheromone tables and further forward it in their broadcasts. This pheromone is called as Virtual pheromone. In proactive ant sampling all source nodes of communication session will periodically broadcast the proactive forward ants. These ants chooses a next node randomly and builds a new path. For choosing the next node they consider both regular and virtual pheromone and they leave the previous routes and follows the routes which were derived in pheromone diffusion phase. After reaching the destination proactive forward ant becomes proactive backward ant and returns to the source.

Failures are managed by using some reactive mechanisms, such as local route repair and warning messages. In local route repair mechanism repair forward ants are sent from the failed node to source node of communication session. They are limited up to particular number of hops. When the number of hops limit is reached then these ants will be discarded. Unicast warning messages are sent from the failed node to the source node. After broadcasting the repair ants also if packets arrive at the broken link then these messages are used for providing the link failure notification.

AODV [2] is a on-demand routing algorithm used in adhoc networks. When it is needed to establish a new route it broadcasts Route Request message (RREQ). On the way RREQ message creates temporary routing table entries and if the receiver or a optimal route is found then that path is made available and a unicast route reply (RREP) message is sent to sender. RREP message creates routing table entries in the intermediate nodes along its path to source node.

\section{LITERATURE SURVEY}

Annapurna P Patil, K Rajanikant, Rakshith H P [4] have compared AntHocNet and AODV using metrics Packet Delivery Ratio, jitter, average end to end delay, throughput, queuing delay, convergence time. They have chosen QualNet-5.0 for simulation. Average End to End delay has increased for AODV with the increasing speeds and number of nodes. For huge number of nodes and high speeds AntHocNet outperformed AODV in case of jitter because it is a multi path routing protocol. Convergence time also increased at high speeds and node densities. AntHocNet outperforms AODV in average packet delivery ratio because AntHocNet uses different ants and for decreasing the packet drops many shorter distant routes are calculated. AntHocNet does not work well for throughput. The average queuing delay is less for AntHocNet with varying pause time. They suggested that for improving AntHocNet the protocol has to be enhanced by finetuning the control packet overhead and by introducing the priority concept.

MaahiAmit, Khemchandani, B. W. Balkhandeanalyzed the performance of AntHocNet with DSR, and AODV. At different pause times and speeds lost packet ratio and normalized routing 
International Journal on Cybernetics \& Informatics (IJCI) Vol. 4, No. 2, April 2015

overhead are calculated by varying number of nodes for different pause times and speeds [5]. AntHocNet is consistent and stable when compared with DSR and AODV because AntHocNet is having high normalized routing overhead. AntHocNet performs well at high data rates, speeds and more number of nodes when considering the Packet loss ratio. DSR and AODV are not suitable for large networks with high mobility of nodes.

S. S. Vasekar, Neelam S. Labhade [6] have evaluated routing algorithms performance for video signal transmission based on ant colony optimization for adhoc networks. Evaluation of video transmission is done using AntHocNet and the results are compared with AODV and DSDV protocols. According to them due to having high throughput and packet delivery ratio AntHocNet is better for video transmission. AntHocNet uses a proper route maintenance mechanism, so when compared with AODV and DSDV end to end delay is less for AntHocNet. AntHocNet is better than AODV and DSDV in terms of Peak signal to noise ratio(PSNR) and Latency.

S.B. Wankhade and M.S. Ali investigated Ant based routing research trends. They have identified Quality of service and routing issues [7]. Because of the dynamic nodes and limited resources current routing algorithms are not sufficient. Ant based algorithms use Fuzzy rule-based systems for reducing these problems. Fuzzy rule-based systems does not exhibit the unstability in a dynamic environment, they also manage the link failures in an efficient way.

Ant-E [8] is a on demand routing protocol and it uses Blocking Expanding Ring Search (Blocking-ERS) for improving the reliability.Reliability is increased by reducing the overhead and resending the data locally. If destination node is not encountered then Blocking-ERS does not approach source node for broadcasting again, intermediate node will take care about the rebroadcasting. This algorithm is used for solving the optimization problems which are complex.

Ant colony based Multi-path QoSaware Routing (AMQR) protocol establishes a number of disjoint paths [9]. Source node establishes a topology by grouping the information of the paths followed by ants. Depending on this information source calculates various link disjoint paths. Using these paths it will send the data. Pheromone information is renewed by data packets.

Swarm-based Distance Vector Routing (SDVR) [10] is an implementation of AntNet. SDVR uses many pheromone tables, each table is allocated for a QOS parameter and all tables are grouped when it is the time for taking a decision. SDVR is suitable for small networks than AODV. FuzzyAntNet follows optimized fuzzy systems and swarm intelligence. It is scalable to modifications in network and it can identify the best route from sender to receiver which avoids the traffic and minimum delay with high bandwidth.

Adaptive Fuzzy Ant-based Routing(AFAR) algorithm [11] builds links between the nodes. Nodes exchange the path information for updating the pheromone tables. By taking the existing network state into account and the previous information new paths are established considering fuzzy logic techniques.

Ant-AODV is a hybrid routing protocol. Ant-AODV can minimize the end to end delay than AODV [12]. AODV is used for reactive mechanism where as Ant algorithms performs the proactive part. Aim of ant routing algorithm is to identify the routes in a minimum time there by reducing end to end delay. Ant-AODV is appropriate for real time applications because minimization of latency in identifying the route is done in Ant-AODV. This protocol uses route error messages to tell the remaining nodes about the path break.HELLO messages tells about the neighbor's presence.

Ant-DSR [13] is a on demand routing protocol in which paths are continuously checked using a cache. The routes are continuously modified when new routes are encountered. Ant-DSR is 
International Journal on Cybernetics \& Informatics (IJCI) Vol. 4, No. 2, April 2015

having two phases, discovering the route and maintaining the route. Forward ants discover the new routes, backward ants will inform source node about the information that is gathered by forward ants.

Ant colony optimized routing protocol(ACOR)[14] is based on swarm intelligence. In this protocol to obtain the best route path maintenance is done periodically. Refreshing of the path is done regularly for adapting the topological changes.

\section{Simulation}

\subsection{Simulation Environment}

In this work for analyzing the performance of AntHocNet protocol, network simulator NS-2.34 is used. Number of nodes considered is 50, Maximum number of connections is 8 , simulation time is $200 \mathrm{sec}$ and the area of simulation is $500 \times 500(\mathrm{~m} \mathrm{x} \mathrm{m})$. The nodes are mobile and the links are wireless. Each node considered as communication end-point is host. Throughput is calculated at different simulation times from 20 to 200 , at data rates $3 \mathrm{Mbps}$, $5 \mathrm{Mbps}$, $10 \mathrm{Mbps}$ and the metrics packet delivery ratio, average end to end delay, loss rate, average jitter are calculated by considering the data rates as 3,5,10 Mbps and varying the speeds as 5,10,20 m/sec at pausetimes $30,100 \mathrm{sec}$.

\subsection{Metrics}

The following metrics are calculated.

- Throughput :Throughput is the number of packets that are received in a particular time, i.e. the rate at which a network receives data.

- Packet Delivery Ratio :Packet delivery ratio is the ratio of number of received data packets to number of sent packets.

- Loss rate :Ratio of dropped packets to number of sent packets is known as Packet loss rate.

- Jitter :The difference of time between the packets arrival is called as jitter.

- End to End delay :Time involved in delivery of data packets from source node to destination node is the End to End delay.

\subsection{Simulation Results and Analysis}

Number of nodes - 50, Pause time - $0 \mathrm{sec}$, Speed - $20 \mathrm{~m} / \mathrm{sec}$ 
International Journal on Cybernetics \& Informatics (IJCI) Vol. 4, No. 2, April 2015

\subsubsection{Graphs for Throughput}

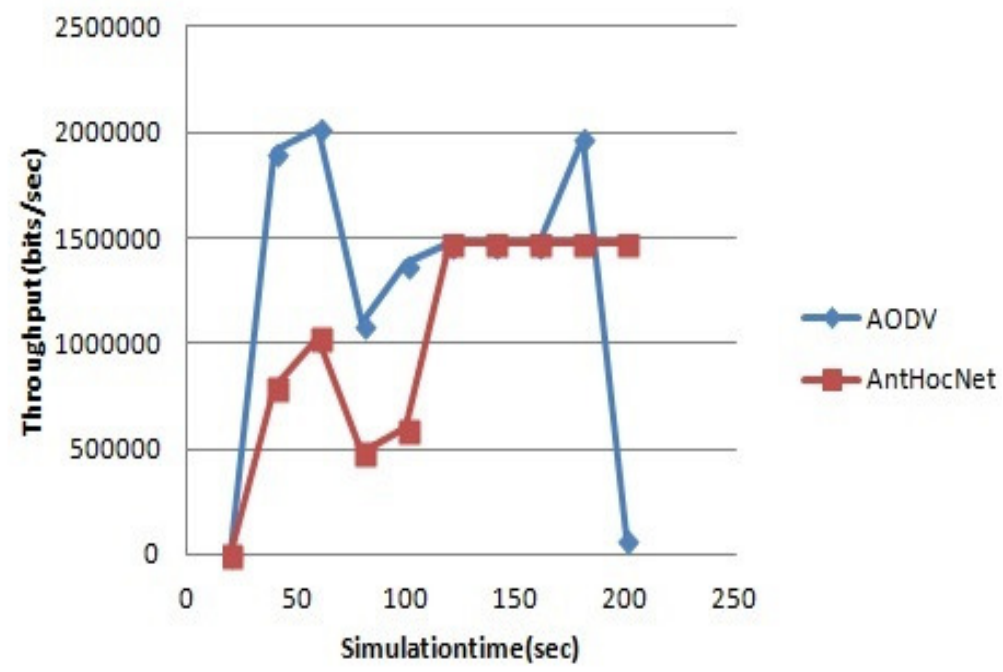

Fig 1:AODV and AntHocNetThroughput at 3Mbps

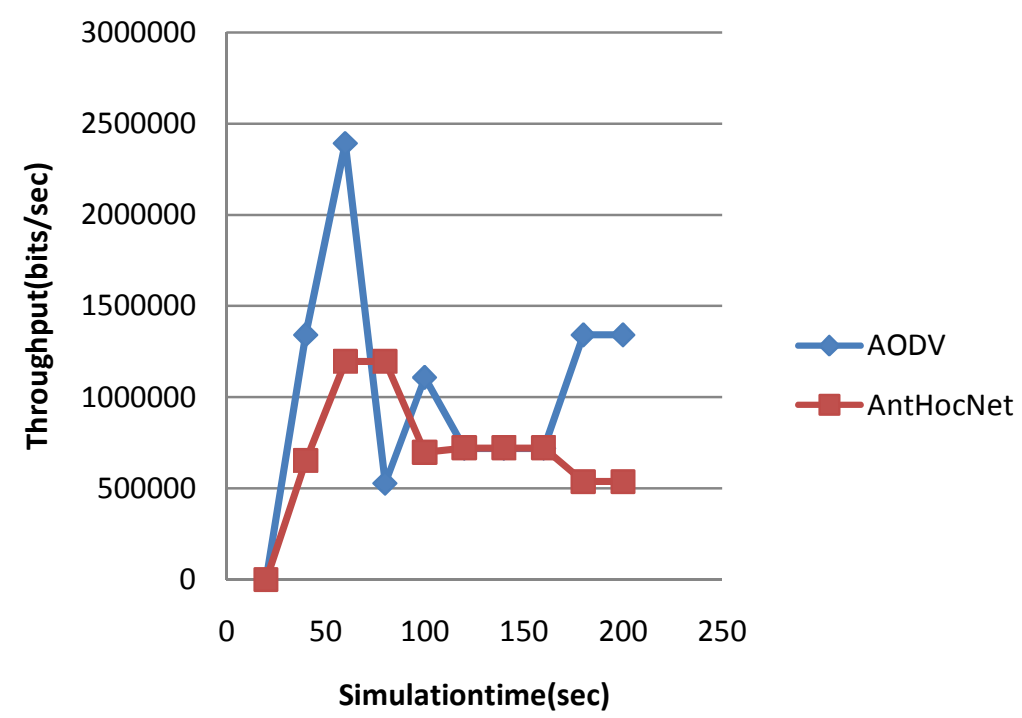

Fig 2 : AODV and AntHocNet Throughput at $5 \mathrm{Mbps}$ 
International Journal on Cybernetics \& Informatics (IJCI) Vol. 4, No. 2, April 2015

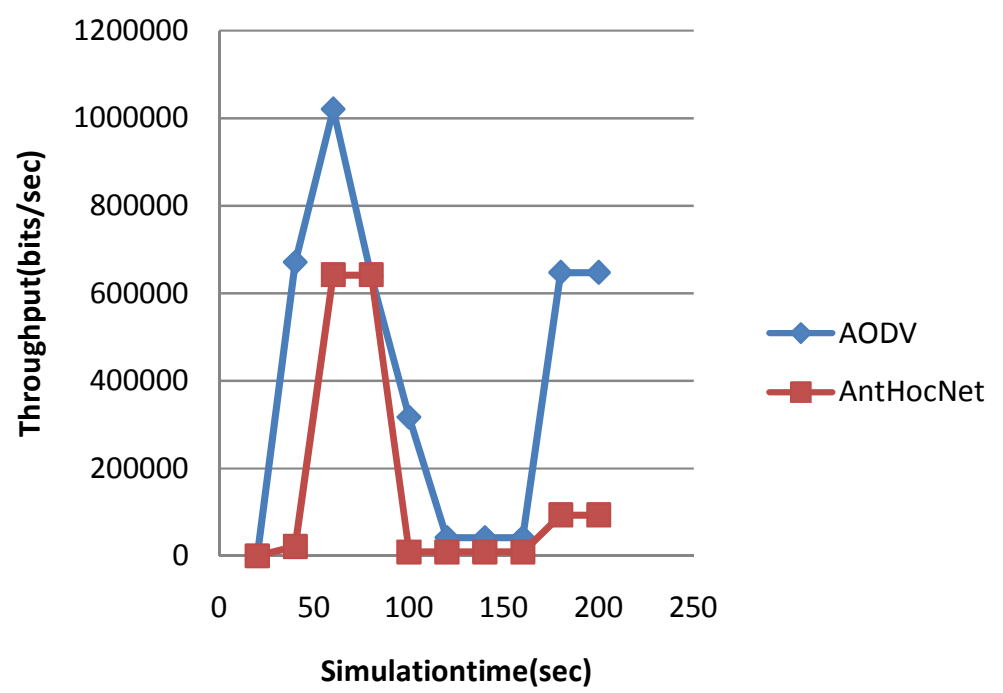

Fig 3 :AODV and AntHocNetThroughput at $10 \mathrm{Mbps}$ Number of nodes - 50, Data rate - 3 Mbps

Table 1 : Packet delivery ratio at different pausetimes and speeds

\begin{tabular}{|l|l|l|l|}
\hline \multirow{2}{*}{$\begin{array}{c}\text { Pause time } \\
(\mathbf{s e c})\end{array}$} & $\begin{array}{l}\text { Speed } \\
(\mathbf{m} / \mathbf{s e c})\end{array}$ & \multicolumn{2}{|l|}{ Packet delivery ratio } \\
\cline { 2 - 4 } & 5 & AODV & AntHocNet \\
\hline 30 & 10 & 0.11299 & 0.10499 \\
\cline { 2 - 4 } & 20 & 0.14142 & 0.0702 \\
\hline \multirow{3}{*}{100} & 5 & 0.14531 & 0.0529 \\
\cline { 2 - 4 } & 10 & 0.12211 & 0.0828 \\
\cline { 2 - 4 } & 20 & 0.13409 & 0.0517 \\
\hline
\end{tabular}

Number of nodes - 50, Data rate - 5 Mbps

Table 2: Packet delivery ratio at different pausetimes and speeds

\begin{tabular}{|l|l|l|l|}
\hline \multirow{2}{*}{$\begin{array}{l}\text { Pause time } \\
\text { (sec) }\end{array}$} & $\begin{array}{l}\text { Speed } \\
(\mathbf{m} / \mathbf{s e c})\end{array}$ & \multicolumn{2}{|l|}{ Packet delivery ratio } \\
\cline { 2 - 4 } & 5 & AODV & AntHocNet \\
\cline { 2 - 4 } & 10 & 0.0956 & 0.0705 \\
\cline { 2 - 4 } & 20 & 0.0889 & 0.0451 \\
\hline \multirow{3}{*}{100} & 5 & 0.0848 & 0.0297 \\
\cline { 2 - 4 } & 10 & 0.0812 & 0.0727 \\
\cline { 2 - 4 } & 20 & 0.0796 & 0.0376 \\
\hline
\end{tabular}

Number of nodes - 50, Data rate - $10 \mathrm{Mbps}$ 
International Journal on Cybernetics \& Informatics (IJCI) Vol. 4, No. 2, April 2015

Table 3 : Packet delivery ratio at different pausetimes and speeds

\begin{tabular}{|l|l|l|l|}
\hline \multirow{2}{*}{$\begin{array}{c}\text { Pause time } \\
\text { (sec) }\end{array}$} & \multirow{2}{*}{$\begin{array}{l}\text { Speed } \\
(\mathbf{m} / \mathbf{s e c})\end{array}$} & \multicolumn{3}{|l|}{ Packet delivery ratio } \\
\cline { 3 - 4 } & 5 & AODV & AntHocNet \\
\cline { 2 - 4 } & 10 & 0.0476 & 0.0191 \\
\cline { 2 - 4 } & 20 & 0.0541 & 0.03 \\
\hline \multirow{3}{*}{100} & 5 & 0.0547 & 0.02 \\
\cline { 2 - 4 } & 10 & 0.0472 & 0.026 \\
\cline { 2 - 4 } & 20 & 0.0628 & 0.0264 \\
\hline
\end{tabular}

Number of nodes-50, Data rate-3 Mbps

Table 4 : Loss Rate at different pausetimes and speeds

\begin{tabular}{|c|c|c|c|}
\hline \multirow{2}{*}{$\begin{array}{l}\text { Pause time } \\
\text { (sec) }\end{array}$} & \multirow{2}{*}{$\begin{array}{l}\text { Speed } \\
(\mathrm{m} / \mathrm{sec})\end{array}$} & \multicolumn{2}{|c|}{ Loss Rate } \\
\hline & & AODV & AntHocNet \\
\hline \multirow[t]{3}{*}{30} & 5 & 0.88701 & 0.89501 \\
\hline & 10 & 0.85857 & 0.9298 \\
\hline & 20 & 0.85469 & 0.94713 \\
\hline \multirow[t]{3}{*}{100} & 5 & 0.87789 & 0.91721 \\
\hline & 10 & 0.8659 & 0.94482 \\
\hline & 20 & 0.86527 & 0.89832 \\
\hline
\end{tabular}

Number of nodes-50, Data rate-5 Mbps

Table 5 : Loss Rate at different pausetimes and speeds

\begin{tabular}{|l|l|l|l|}
\hline \multirow{2}{*}{$\begin{array}{c}\text { Pause time } \\
(\mathbf{s e c})\end{array}$} & $\begin{array}{l}\text { Speed } \\
(\mathbf{m} / \mathbf{s e c})\end{array}$ & Loss Rate & \\
\cline { 2 - 4 } & 5 & AODV & AntHocNet \\
\hline \multirow{3}{*}{0} & 5 & 0.9044 & 0.92949 \\
\cline { 2 - 4 } & 10 & 0.9111 & 0.95491 \\
\cline { 2 - 4 } & 20 & 0.9151 & 0.97026 \\
\hline \multirow{3}{*}{100} & 5 & 0.9187 & 0.9272 \\
\cline { 2 - 4 } & 10 & 0.9204 & 0.9624 \\
\cline { 2 - 4 } & 20 & 0.9106 & 0.9444 \\
\hline
\end{tabular}

Number of nodes-50, Data rate-10Mbps

Table 6 : Loss Rate at different pausetimes and speeds

\begin{tabular}{|l|l|l|l|}
\hline \multirow{2}{*}{$\begin{array}{c}\text { Pause time } \\
(\mathbf{s e c})\end{array}$} & $\begin{array}{l}\text { Speed } \\
(\mathbf{m} / \mathbf{s e c})\end{array}$ & Loss Rate & \\
\cline { 3 - 4 } & 5 & AODV & AntHocNet \\
\hline \multirow{3}{*}{30} & 10 & 0.9524 & 0.9809 \\
\cline { 2 - 4 } & 20 & 0.9458 & 0.9699 \\
\hline \multirow{3}{*}{100} & 5 & 0.9453 & 0.9799 \\
\cline { 2 - 4 } & 10 & 0.9528 & 0.9739 \\
\cline { 2 - 4 } & 20 & 0.9372 & 0.9736 \\
\hline
\end{tabular}

Number of nodes-50, Data rate-3 Mbps 
International Journal on Cybernetics \& Informatics (IJCI) Vol. 4, No. 2, April 2015

Table 7 : Jitter at different pausetimes and speeds

\begin{tabular}{|l|l|l|l|}
\hline \multirow{2}{*}{$\begin{array}{c}\text { Pause time } \\
(\mathbf{s e c})\end{array}$} & $\begin{array}{l}\text { Speed } \\
(\mathbf{m} / \mathbf{s e c})\end{array}$ & Jitter $(\mathbf{s e c})$ & AntHocNet \\
\cline { 2 - 4 } & 5 & AODV & 10.5431 \\
\cline { 2 - 4 } & 10 & 4.96264 & 43.9245 \\
\cline { 2 - 4 } & 20 & 5.24198 & 34.861 \\
\hline \multirow{3}{*}{100} & 5 & 4.48386 & 19.18796 \\
\cline { 2 - 4 } & 10 & 4.65202 & 23.74592 \\
\cline { 2 - 4 } & 20 & 4.42073 & 8.07373 \\
\hline
\end{tabular}

Number of nodes-50, Data rate-5 Mbps

Table 8 : Jitter at different pausetimes and speeds

\begin{tabular}{|l|l|l|l|}
\hline \multirow{2}{*}{$\begin{array}{c}\text { Pause time } \\
(\mathbf{s e c})\end{array}$} & $\begin{array}{l}\text { Speed } \\
(\mathbf{m} / \mathbf{s e c})\end{array}$ & Jitter (sec) \\
\cline { 2 - 4 } & 5 & AODV & AntHocNet \\
\cline { 2 - 4 } & 10 & 2.8363 & 6.729 \\
\cline { 2 - 4 } & 20 & 2.482 & 5.996 \\
\hline \multirow{3}{*}{100} & 5 & 2.0025 & 1.1995 \\
\cline { 2 - 4 } & 10 & 20.1815 & 25.8462 \\
\cline { 2 - 4 } & 20 & 16.7789 & 11.756 \\
\hline
\end{tabular}

Number of nodes-50, Data rate-10 Mbps

Table 9 : Jitter at different pausetimes and speeds

\begin{tabular}{|l|l|l|l|}
\hline \multirow{2}{*}{$\begin{array}{c}\text { Pause time } \\
(\mathbf{s e c})\end{array}$} & $\begin{array}{l}\text { Speed } \\
(\mathbf{m} / \mathbf{s e c})\end{array}$ & Jitter $(\mathbf{s e c})$ & \\
\cline { 2 - 4 } & 5 & AODV & AntHocNet \\
\cline { 2 - 4 } & 5 & 4.5214 & 13.4077 \\
\cline { 2 - 4 } & 10 & 2.4054 & 11.7007 \\
\hline \multirow{3}{*}{100} & 5 & 3.1299 & 13.9545 \\
\cline { 2 - 4 } & 10 & 9.1517 & 13.5595 \\
\cline { 2 - 4 } & 20 & 4.6306 & 10.4929 \\
\hline
\end{tabular}

Number of nodes-50, Data rate-3 Mbps

Table 10 : End to End delay at different pausetimes and speeds

\begin{tabular}{|l|l|l|l|}
\hline \multirow{2}{*}{$\begin{array}{c}\text { Pause time } \\
(\mathbf{s e c})\end{array}$} & $\begin{array}{l}\text { Speed } \\
(\mathbf{m} / \mathbf{s e c})\end{array}$ & \multicolumn{2}{|l|}{ End to End delay $(\mathbf{s e c})$} \\
\cline { 2 - 4 } & 5 & AODV & AntHocNet \\
\cline { 2 - 4 } & 10 & 1.2499 & 1.11352 \\
\cline { 2 - 4 } & 20 & 0.97092 & 1.9159 \\
\hline \multirow{3}{*}{100} & 5 & 0.96282 & 1.58094 \\
\cline { 2 - 4 } & 10 & 1.18112 & 1.61025 \\
\cline { 2 - 4 } & 20 & 0.96052 & 1.61548 \\
\hline
\end{tabular}

Number of nodes-50, Data rate-5 Mbps 
Table 11 : End to End delay at different pausetimes and speeds

\begin{tabular}{|l|l|l|l|}
\hline \multirow{2}{*}{$\begin{array}{c}\text { Pause time } \\
(\mathbf{s e c})\end{array}$} & $\begin{array}{l}\text { Speed } \\
(\mathbf{m} / \mathbf{s e c})\end{array}$ & End to End delay (sec) \\
\cline { 2 - 4 } & 5 & AODV & AntHocNet \\
\hline \multirow{3}{*}{30} & 10 & 0.7754 & 1.0546 \\
\cline { 2 - 4 } & 20 & 0.9511 & 1.4881 \\
\hline \multirow{3}{*}{100} & 5 & 0.9907 & 2.0098 \\
\cline { 2 - 4 } & 10 & 1.0778 & 1.291 \\
\cline { 2 - 4 } & 20 & 1.0596 & 2.1014 \\
\hline
\end{tabular}

Number of nodes-50, Data rate-10 Mbps

Table 12 : End to End delay at different pausetimes and speeds

\begin{tabular}{|l|l|l|l|}
\hline \multirow{2}{*}{$\begin{array}{c}\text { Pause time } \\
(\mathbf{s e c})\end{array}$} & \multirow{2}{*}{$\begin{array}{l}\text { Speed } \\
(\mathbf{m} / \mathbf{s e c})\end{array}$} & End to End delay (sec) \\
\cline { 2 - 4 } & 5 & AODV & AntHocNet \\
\cline { 2 - 4 } & 10 & 0.9826 & 1.5264 \\
\cline { 2 - 4 } & 20 & 0.8417 & 1.5507 \\
\hline \multirow{3}{*}{100} & 5 & 0.8532 & 1.4273 \\
\cline { 2 - 4 } & 10 & 1.007 & 1.87051 \\
\cline { 2 - 4 } & 20 & 0.7162 & 2.0072 \\
\hline
\end{tabular}

\section{DISCUSSION}

Figures 1, 2 and 3 represents the evaluation of throughput at different data rates, speed $20 \mathrm{~m} / \mathrm{sec}$, pausetime $0 \mathrm{sec}$. Figure 1 illustrates that AODV's throughput is higher than AntHocNet at $3 \mathrm{Mbps}$. At data rate 5 Mbps AntHocNet's throughput is less than AODV as shown in Figure 2. From Figure 3 it is concluded that AODV throughput has outperformed AntHocNet at $10 \mathrm{Mbps}$.

Packet delivery ratio, Loss rate, End to End delay and jitter are calculated at data rates 3, 5, 10 Mbps, speed is varied as 5,10,20 m/sec and pause time is taken as 30, $100 \mathrm{sec}$. From Tables 1, 4, 7, 10 it is inferred that at data rate 3 Mbps AODV performs better than AntHocNet in terms of packet delivery ratio and AntHocNet exhibits higher loss rate, jitter and, end to end delay.

AODV packet delivery ratio is better than AntHocNet and AntHocNet is having more loss rate, jitter, and end to end delay at data rate $5 \mathrm{Mbps}$ as shown in Tables 2, 5, 8, 11.

At data rate 10 Mbps AntHocNet packet delivery ratio is less than AODV and loss rate, end to end delay, jitter are more than AODV and are presented in Tables 3, 6, 9, 12.

\section{CONCLUSION}

In this work two routing protocols are compared namely Adhoc On demand Distance Vector Routing protocol and AntHocNet. The parameters pause time and speed are kept constant and they are set to $0 \mathrm{sec}$ and $20 \mathrm{~m} / \mathrm{sec}$ respectively and the data rates are considered as $3 \mathrm{Mbps}, 5$ Mbps and $10 \mathrm{Mbps}$ respectively. We have calculated the throughput at different simulation times and it is observed that AODV outperforms AntHocNet at 3, 5 and 10 Mbps. At data rates 3, 5, 10 Mbps by varying pause time as 30,100 sec and speed as 5, 10, $20 \mathrm{~m} / \mathrm{sec}$ AODV performs better than AntHocNet in terms of packet delivery ratio, throughput and AntHocNet is having high loss 
rate, end to end delay and jitter. So, it is concluded that AODV is performance is better than AntHocNet in the simulations considered.

\section{REFERENCES}

[1] Frederic Ducatelle, (2007) "Adaptive Routing in Wireless Adhoc Multi Hop Networks", Switzerland.

[2] C. E. Perkins, E. M. Belding Royer and S. Das, (2003) "Adhoc On Demand Distance Vector Routing", RFC 3561, Available at :www.ietf.org/rfc/rfc3561.txt.

[3] Gianni Di Caro, Frederick Ducatelle and Luca Maria Gambardella, (2005) " An Adaptive natutreinspired algorithm for routing in Mobile Adhoc networks", European Transaction on Telecommunications, pp. 16:443 - 455.

[4] Annapurna P Patil, K Rajanikant, Rakshith H P, (2012) "Analyzing the performance of AntHocNet protocol forMANETS", International Journal of Computer Applications(0975-8887), Vol. 57, No. 5, pp. 20-25.

[5] MaahiAmitKhemchandani, Prof. B. W. Balkhande, (2014) "Comparitive analysis of AntHocNet, AODV, DSRRoutingProtocols for improving Loss Packet Delivery Factor", International Journal of Computer Science andInformation Technologies, Vol. 5 (5), pp.6478-6482.

[6] Neelam S. Labhade, S. S. Vasekar, (2014) "Performance evaluation of AODV, DSDV and AntHocNet in videotransmission", IJARCET - International Journal of Advanced Research in Computer Engineering \& Technology,Vol. 3, Issue 5, pp. 1934-1939.

[7] S. B. Wankhade and M. S. Ali, (2012) "Recent Trends in Ant Based Routing Protocols for MANET", InternationalJournal of Advances in Engineering \& Technology, Vol. 4, Issue 1, pp. 405-413.

[8] SrinivasSethi\&SibaK.Udgata, (2002) "The Efficient Ant Routing Protocol for MANET", In International Journal onComputer Science and Engineering, Vol. 2, No.7, pp. 2414-2420.

[9] L. Liu \& G. Feng, (2005) "A Novel ant colony based QoS aware routing algorithm for MANETS", In proceedings ofFirst International Conference on advances in Natural Computation, Changsha(China), Volume 3612, pp. 457-466.

[10] R. Ashokan, A. M. Natarajan\& C. Venkatesh, (2008) "Ant based Dynamic Source Routing Protocol to SupportMultiple Quality of Service Metrics in Mobile Adhoc Networks", In International Journal of Computer Scienceand Security, Volume 2, No.3, pp.48-56.

[11] S. J. Mirabedini, M. Teshnehlab, M. H. Shenasa, Ali Moraghar\& A. M. Rahmani,(2008) "AFAR Adaptive fuzzyant based routing for communication networks", In Journal of Zhejiang University Science, Volume 9, No. 12,pp.1666-1675.

[12] S. Marwaha, C. Khong and T. Srinivasan,(2002) "Ant-AODV : Mobile agents based routing protocol for mobileadhocnetworks", In Proceedings of IEEE Global Telecommunications Conference, GLOBECOM,Taipei(Taiwan), Vol. 1, pp. 163-167.

[13] M. Aissani, M. Fenouche, H. Sadour and A. Mellouk, (2007) "Ant-DSR: cache maintainence based routing protocolfor mobileadhocnetworks", AICT 2007- The Third Advanced International Conference inTelecommunications, Morne, pp. 35-35.

[14] Dweepnagarg, Parthgohil, (2012) "Ant Colony Optimized Routing For Mobile Adhoc Networks", International Journal of Smart Sensors and Adhoc Networks, Vol 2. 